\title{
Magnetic Resonance Imaging of the Whole Body with and without Contrast
}

National Cancer Institute

\section{Source}

National Cancer Institute. Magnetic Resonance Imaging of the Whole Body with and

without Contrast. NCl Thesaurus. Code C137921.

Magnetic resonance imaging of the whole body with and without the use of a contrast agent to enhance the image. 